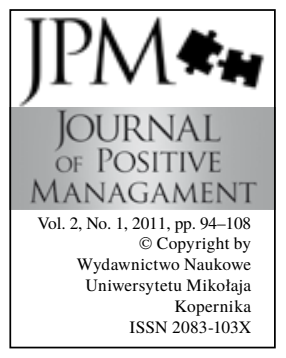

\title{
THE POSITIVE POTENTIAL OF HIGH-PERFORMANCE ORGANIZATION
}

\author{
Przemysław Zbierowski ${ }^{\mathrm{a}}$ \\ ${ }^{\text {a } U n i v e r s i t y ~ o f ~ E c o n o m i c s ~ i n ~ K a t o w i c e, ~ K a t o w i c e, ~ P o l a n d, ~}$ \\ e-mail: przemek@zbierowski.com
}

\begin{abstract}
The paper presents an attempt to empirically answer the question about the source of success for small and medium enterprises (SME) made on the basis of two recently developed theoretical concepts namely High Performance Organization (HPO) and Positive Organizational Scholarship (POS). The aim of the study is to combine the model of HPO with the achievements of POS and to indicate positive characteristics of highly effective organizations - positive phenomena that drive an organization toward success. Data Envelopment Analysis (DEA) was used as the method for measuring relative efficiency in twelve SMEs. It appears from the data that there are different profiles of efficiency, thus there is no such thing as a universal configuration of positive characteristics always leading to high efficiency.
\end{abstract}

Keywords: High Performance Organization, Positive Organizational Scholarship, performance and efficiency measurement, Data Envelopment Analysis

Paper Type: Research paper

\section{Introduction}

One of the breakthroughs that has been made in the teachings of the organization and still continues to be made is the way of looking at the effectiveness of an organization, and thus a new approach to study, not only the organizational effectiveness, but also the organization itself. Traditional research approaches assumed the study of relationship between variables concerning the organization, both internal and external. These variables affected in part the efficiency of organization, which allowed researchers to propose practical recommendations for strategies, techniques and management methods that positively affected efficiency. In recent years there has been a change in approach from the study of all organizations to focus on exceptional organizations and treat them as a reference point. This change in approach was also supplemented by the introduction of new research methods and statistical tools that are tailored to it.

The question about high efficiency of the organization has been asked for many years in the study of organization and management. Currently, this trend gained additional momentum with the consolidating of the concept of high 
performance organization (HPO). Thus created field of research aims to identify characteristics of organizations that achieve high efficiency, what strategies, methods and management methods they use. Another field of study that was discovered in the past decade and which corresponds to it is called a positive organizational scholarship (POS). It proposes a new point of view and treatment of the organization as a "mystery" or "miracle" to be admired, rather than as a problem that must be resolved. Without ignoring traditional theories, it emphasizes "positive" phenomena, such as nobility, vitality, seriousness leading to perfection, transcendence, positive difference, remarkable efficiency, positive spirals of bloom. These trends will serve as help in an attempt to empirically answer the question about the source of success for small and medium enterprises.

\section{Positive Organizational Scholarship}

POS was established in 2003 with the publication of the work that lay foundations for this field (Cameron, Dutton and Quinn, 2003). POS offers a new look at old problems, and in fact it goes further, proposing to forget about problems that require solutions, and instead focus on the organization as a "mystery" or "miracle" that we should admire. This new perspective can bring benefits in terms of better understanding of organizational phenomena and discovering new, previously invisible to researchers equipped with traditional approaches and techniques.

Positive science of organization takes its main inspiration from positive psychology, developed by the former president of the American Psychological Association Martin E. Seligman in 1998. Positive psychology proposes a different from the traditional one perspective without replacing traditional approaches, but attempting to supplement them. It diverts attention from what people are missing on what they have, highlighting the strengths of human nature, allowing them to build a successful life, flourish and be happy (Seligman, 2002).

The field closest to the positive science of organization and the most contributing to its creation and development is the development of organization, in particular the appreciative approach. This is a research trend in the development of an organization that seeks to harness the positive aspects of human personality in order to strengthen the organization's ability to change and develop. This is a practical philosophy, which is based on the assumption that an organization is a "mystery" or "miracle" to be admired, not a problem to be solved (Cooperrider and Srivastava, 1987).

A positive science of the organization is a kind of new philosophy of organization. While it does not reject the organizational and social phenomena, such as greed, selfishness, manipulation, lack of confidence or anxiety, it tries to emphasize "positive" phenomena, such as gratitude, appreciation, cooperation, integrity, vitality, a sense of meaning, credibility, resilience, wisdom, loyalty, 
THE POSITIVE POTENTIAL

Przemysław Zbierowski respect and honesty. A positive science of the organization is a new approach both ontological and epistemological; it sheds new light on what the organization is and how to learn it. It is not a single theory, it is more of a viewpoint that emphasizes positive and dynamic social and organizational phenomena, extending attention to conditions (processes, capabilities, structures, methods), motivation (selflessness, altruism) and results (vitality, a sense of meaning, happiness, good relationships) associated with positive phenomena. A positive science of the organization also stresses the dynamics with special emphasis on non-linear positive change "positive spirals".

Perhaps the most comprehensive approach to Positive Organizational Scholarship was presented by Stankiewicz and the team of the Department of General Management of Nicolaus Copernicus University in Torun (Stankiewicz, 2010). The presented model and conducted research reveals complex relationships between Positive Organizational Potential, its antecedents (internal and external context), Positive Organizational Culture, Positive Organizational Climate, Positive Behaviors and firm's performance.

To better recognize positive phenomena we can classify them into a matrix of levels and areas of a positive organization science (Table 1). Cameron, Dutton, Quinn and Wrzesniewski (2003) suggest the existence of three levels of occurrence of positive effects: individual, organization and society. Also they propose to consider the question whether the relationship between these phenomena observed at one level can be extrapolated to another level, which in particular relates to the organizational performance of individual phenomena. A positive science of the organization also focuses on three main areas of occurrence of positive developments: the causes, determinants and consequences of positivity. Intersection of levels with regions provides a better insight into the nature of positive developments. However, this matrix can be read with the reservation that it is not complete; it does not utilize their richness.

\section{High Efficiency Organizations}

Over the past few years, there appeared in the literature many models of highperformance organizations (Holbeche, 2005; de Waal, 2005; de Waal, 2006). In particular, several factors are related to effectiveness: (1) project of organization, (2) strategy, (3) process management, (4) leadership, (5) long-term orientation, (6) continuous improvement, (7) organizational culture, (8) orientation to the outside.

The aim of this study is to combine this model with the achievements of positive science of management and to indicate positive characteristics of highly effective organizations - positive phenomena that drive an organization toward success. We can conclude that high efficiency is in itself positive, because it is a reflection of organizational excellence. If this is the case then the positive science 
of management should have high explanatory power in analyzing the processes of achieving and maintaining high efficiency and good characteristics should serve as its foundation.

Analysis of different models of highly efficient organizations allows us to observe a specific order among their characteristics. It can be assumed that the order is established by natural forces of an organization. Another interesting observation is that most of the characteristics of highly effective organizations are characteristics from the field of a positive management science, such as empowerment, freedom of action, trust and cooperation.

Using the classical model proposed by Galbraith (1995) we can propose a highly effective organizational model based on how the six main elements fit together: strategy, culture, people, structure, tasks and systems (Table 1). In this model, each component has a form of providing fit, which in turn leads to achieving high performance in a positive context.

\begin{tabular}{|l|l|}
\hline $\begin{array}{c}\text { Element } \\
\text { of organi- } \\
\text { zation } \\
\text { project }\end{array}$ & \multicolumn{1}{c|}{ Element's features } \\
\hline Strategy & $\begin{array}{l}\text { Outstanding (clear and challenging) vision - thinking in the future tense, vision- } \\
\text { ary goals posing challenges }\end{array}$ \\
\hline Culture & $\begin{array}{l}\text { Culture relationships based on trust, social integration, improvisation (innovation, } \\
\text { wide range of freedom to experiment, ability to change) }\end{array}$ \\
\hline People & $\begin{array}{l}\text { Attracting exceptional (positively, internally motivated) people who are strongly } \\
\text { committed, skilled in empowerment, involved in organizational leadership and } \\
\text { citizenship }\end{array}$ \\
\hline Structure & $\begin{array}{l}\text { Flexible structure - functional flexibility, decentralization, deformalization, flat } \\
\text { and simple structure }\end{array}$ \\
\hline Tasks & $\begin{array}{l}\text { The tasks of sharing information and knowledge, continuous improvement of new } \\
\text { processes and products, creating value for stakeholders }\end{array}$ \\
\hline Systems & $\begin{array}{l}\text { Systems of fair remuneration and motivation; open communication, flexible sys- } \\
\text { tems across the whole organization }\end{array}$ \\
\hline
\end{tabular}

Table 1.

Highly effective organization model

Source: own study.

The strategically proposed model recommends for the organization to have a sustainable vision that motivates to make intense effort; on the other hand, the process of creating a strategy must be continuous in order to ensure the organization is capable of fitting outside. Key elements of this process are alertness and thinking in the future tense. An important element is also setting goals that are challenging for the participants of the organization. Culture of highly effective organizations is based on strong core values, however it still leaves a wide scope of discretion, particularly in the field experiment. It is important for culture to focus 
THE POSITIVE POTENTIAL

Przemysław Zbierowski on high performance. This culture is also innovative, open to continuous change and based on trust between participants in an organization and stakeholders. In terms of human resources management it is crucial to attract appropriate and in fact exceptional people to the organization, and consequently skilfully use their potential and develop it, in which a special role is played by modern concepts such as empowerment and coaching.

In building the structure for highly efficient organization it is essential to maintain its simplicity and flatness, which will allow the organization to continually adapt to the environment and other elements of the project. The main tasks of the organization on its way to high efficiency is to build rapport with stakeholders, which is made through the creation of values for them. Other important tasks include sharing the know-how and continuous improvement of existing processes within the organization. Essential systems for high efficiency are systems of remuneration and incentives based on equitable rules, and information technologies providing a platform for innovation and communication within and outside the organization, at the same time designed across the entire organization in such a way that they could easily adapt to the challenges they face.

\section{Data Envelopment Analysis}

Data Envelopment Analysis (DEA), also known as the edge analysis is an extremely effective method for measuring relative efficiency. The unit of analysis in DEA is called a DMU - Decision Making Unit. DMUs can be almost anything: businesses, non-profit institutions, even people. DEA was previously used to measure the effectiveness of bank branches, government agencies, police departments, universities, hospitals, airlines, as well as baseball players. The basic idea of DEA is that the individuals tested are homogeneous, which means they operate under the same conditions, and they use the same inputs to produce the same outputs. Proper identification of inputs and outputs is critical to the effectiveness of the DEA. Such a choice should be complete and include the most important resources and effects of a unit, both quantitative and qualitative.

Traditional measures of efficiency are ratios of outputs (such as income) to the inputs (for example, assets). The higher the output and the lower the entry, the higher the efficiency of operations. In the analysis of the DEA, which takes into account multiple inputs and outputs, the effectiveness of the unit is the quotient of the weighted sum of outputs to the weighted sum of inputs. Traditionally, in measuring effectiveness, the weights are in this case set arbitrarily, usually by means of research. The novelty of DEA lies in the fact that the weights are not known prior to conducting analysis and are not the same for each unit. Instead, they are calculated for each unit in such a way that that they are the most beneficial 
for them. Quotient of the weighted sum of outputs to the weighted sum of inputs is then recalculated so that it is a number between 0 and 1 .

DMUs whose efficiency is 1 are called effective or highly effective (in terms of HPO - High Performance Organization), those with rates lower than 1 are inefficient or less-effective. Highly efficient units make up the "efficiency barrier", which is a line (only in two-dimensional space, assuming there is one input and two outputs) enveloping the lower-efficient DMU. Although units A and D produce a completely different combination of outputs they are both effective.

DEA has enormous potential for application, in addition to measuring the effectiveness it proposes recommendations for units to improve efficiency. This means moving a unit onto the efficiency barrier by minimizing the consumption of inputs or maximizing production outputs. What can be seen is a few practical advantages of data envelopment analysis in comparison with a classic measurement of efficiency. It can measure the effectiveness taking into consideration multiple inputs and outputs simultaneously, they can also be expressed in different units, and even be qualitative data, as the DEA is not a statistical method. It also allows for setting targets to improve efficiency, which can become the basis for a process of formulating strategy, both in terms of setting goals by maximizing outcomes and reducing costs. DEA also identifies individual benchmarks for each inefficient unit. The benchmark units are effective units, and they are at the verge of efficiency, close to the analyzed inefficient unit. The observation of model units allows for benchmarking of the best techniques, methods and practices to improve efficiency. Another practical application of DEA is to establish virtual inputs and outputs. In this manner, the inefficient units receive information as to in which areas their inefficiency manifests itself, and exactly where to make improvements.

\section{Research Hypotheses}

Outstanding vision plays an important role in creating high efficiency. According to some researchers, the impact of outstanding vision on the effectiveness is indirect, since it creates a higher level of commitment to the organization and work. The same authors emphasize that the excellence of vision is particularly important for firms operating in turbulent environments. The vision is outstanding when participants in the organization feel that it is clearly articulated, leaders in the company share this vision and the vision is correct.

The basis for highly efficient organizational cultures is trust. A key feature of trust is that it requires from the subject of trust acceptance of irreducible social sensitivity (lack of immunity) and uncertainty. Organizational trust is the most important for high-performance organizational model. Some organizations produce a greater range of trust than others. Thus generated high level of trust can govern and supervise strategic activities of an organization. There is ample

THE POSITIVE POTENTIAL

Przemysław Zbierowski 
THE POSITIVE POTENTIAL

Przemysław Zbierowski evidence that the confidence level teams and the organization have a strong positive impact on the effectiveness of an organization.

Another element of highly effective organizational cultures is social integration. It is a phenomenon analyzed at different levels (individual, group, organizational and social) and can therefore be taken into account at every level of analysis of positive phenomena. It can also be the cause of both positive phenomena (high-efficiency, prosperity) as well as the consequence of others (education, knowledge). There is ample evidence that social integration affects effectiveness, e.g. indirect impact on the effectiveness of teams, or by creating social capital and common identity.

Involvement in the work refers to the extent to which individuals feel that they are personally "invested" in the work and identified with it and it is traditionally connected with the Protestant work ethic. From the list of positive developments certainly teamwork, empowerment, focus on the customer and open communication positively influence work commitment. The results show that commitment to work has a positive impact on efficiency, commitment to work which not only results in higher efficiency, but also higher levels of job satisfaction, organizational civic behaviour, and better mental health and wellbeing. Commitment to work is closely linked and interdependent with the organizational and managerial dedication.

Another positive phenomenon contained in the "People" component of an organizational project and related to the previously reported three is internal motivation. Researchers of positive science of management have a tendency, perhaps not entirely justified, to strongly associate it with positive motivation, since it comes from a positive nucleus of man and organizations. Internal motivation is closely linked with commitment to work. The adjective "intrinsic" is used to emphasize that this is the motivation for personal achievement and success in the task rather than "external" satisfaction resulting from factors such as additional wages or better working conditions.

In addition to relationships based on trust and social integration another important positive aspect of high-performance culture is improvising. Organizational improvisation is manifested in three main areas: creativity and bricolage - the scope in which individuals are able to create innovative solutions in conditions of limited resources available through re-engaging available resources; their ability to operate and develop in an environment characterized by pressure and stress; and spontaneity and endurance - orientation on action and determination of an individual to achieve goals and immediate problem-solving.

High efficiency requires employment of a specific type of participants in the organization - internally, positively motivated, highly committed and committing themselves to the work and organization. These people on the other hand, require a specific approach in their management, in which empowerment is probably one of the key aspects. Empowerment can be seen from two related perspectives - 
organizational and individual. The first sees empowerment as a set of managerial activities and practices delegating power and control to subordinates - it is in this context a tool of leadership. In the second perspective empowerment is the perception of being fortified. It is also described as a state of mind of empowerment. In this context, empowerment can be considered as a result of actions and behaviour of leaders.

Following the path of positive characteristics of high efficiency it can be said that empowerment requires on the one hand, on the other produces a flat and flexible structure of organization - delegates power and decision-making to lower levels of management. There are at least two major approaches to organizational flexibility - one emphasizing the functional and numerical flexibility, the other focusing on decentralization and deformalization of the structure. Some researchers suggest that the flexible structure requires decentralization of decision making, low level of formalization and a high degree of permeability of boundaries and relations based on cooperation. In particular, functional flexibility appears to be important for organizations where high-efficiency mechanisms, such as strengthening of workers to participate in decision making, enabling them to work in teams, organizational strengthening of sacrifice or tying salary with organizational efficiency.

The next two positive characteristics of high efficiency - sharing information and knowledge, and open communication are among the components of 'task' and 'systems' of project organization, but they are closely related and often cooccur in an organization. Teams and organizations that develop a skill to share information and knowledge have less need to plan, higher level of cooperation, less misunderstanding, lower level of confusion and thus they operate more efficiently. Sharing information may also increase the effectiveness of teams and organizations with a high degree of diversity. Open communication is positively associated with both perceived longevity of the team and satisfaction of its members, and this affects organizational efficiency according to the theory of higher class.

The last of the described positive characteristics of high efficiency is the system of remuneration and promotion, which is adjusted for other factors - is fair and focused on efficiency. The research results indicate that in the environment of high efficiency there is a high pressure for justice, and that the perception of justice can produce a higher level of efficiency. According to the claims of Kalleberg functional flexibility created for high organizational efficiency also requires a link between remuneration of organization members and organizational effectiveness.

This literature review and conceptualization of the model suggest that positive characteristics of high efficiency have a positive impact on individual and organizational effectiveness. This is not surprising, as most part of the
THE POSITIVE POTENTIAL

Przemysław Zbierowski 
THE POSITIVE POTENTIAL

Przemysław Zbierowski characteristics was the subject of theoretical or empirical research as reasons for high efficiency. We can therefore form the following research hypothesis:

H1: Highly efficient organizations have a high level of positive characteristics of high efficiency.

Moreover the cited results indicate that the positive characteristics of high efficiency are interrelated. This is consistent with the theory of organizational fit, according to which only organizations with a specific configuration of traits can be successful. We can therefore come up with another research hypothesis:

H2: Highly efficient organizations have a high level all the positive characteristics of high efficiency.

As the conducted study does not aim to explore the relationships between various positive characteristics of high efficiency and the effectiveness of hypothesis does not seem necessary at this point.

\section{Study Design - variables, measurements and research attempt}

Previously described positive characteristics of high effectiveness and efficiency measure are the inputs and outputs of the DEA model. The excellence of vision was measured using a 3-component measurement, trust by the 8-component tool, social integration by the 9-component modified to measure at the organizational level, commitment to work was measured using a five-component measure that has been verified as a reliable tool in different cultural contexts, organizational commitment was examined using a 4-component scale of the Organizational Commitment Questionnaire, internal motivation was measured by a 6-component measure, improvisation by the scale consisting of 9 ingredients ( 3 on each dimension), empowerment was measured using the 12-component (three components for each dimension) of the Spreitzer's scale. Organizational flexibility as functional flexibility was investigated using a 5-component Kalleberg's scale as decentralization/deformalization by a 4-component scale based on Hatum's and Pettigrew's research, knowledge sharing by the 4-component tool, openness of communication was measured by the scale of 2-components, justice by the 3-component tool. In all of the tools the 7-point Likert's scales were used, where it was necessary tools were adapted for use at the organizational level.

Growth of the company was measured by increased sales, profitability by the ratio of profit per employee, the credibility of the three-component, 5-point scale, the goals of organizations by 5-point self-assessment, innovation was measured on a 3-component, 7-point scale, which was part of the entrepreneurial orientation scale, job satisfaction by 3-component, 7-point Michigan Organizational Assessment Questionnaire (MAOQ). In addition, the age of the organization and its size (number of employees) were used as control variables.

The research assessment consisted of twelve highly efficient small and medium-sized businesses. They have been selected by the ranking of "Business 
Gazelles" conducted by "Puls Biznesu". To be included in the ranking a company must meet the following conditions: run continuously for the past three years, make a profit for the past three years, have a turnover of not less than 3 million zloty and not more than 200 million, increase the turnover each year over the last three years, and have a good company record. As the attempt to apply the method of data envelopment analysis must be homogeneous, the study concerned companies from one industry - trade in steel products, which have a similar type of activity. All 55 such companies were included in the ranking of business gazelles, questionnaires were sent to all by e-mail, followed by a phone call. Twelve completed questionnaires were delivered, yielding a response rate of $22 \%$. The collected empirical material was analyzed using data envelopment analysis and xIDEA software by ProdTools, some calculations were also performed using the SPSS package. Additional telephone calls from companies that completed questionnaires were conducted to verify the data.

\section{Research results}

Data Envelopment Analysis (DEA) is a nonparametric, benchmarking method for analyzing models with multiple inputs and outputs, which will identify the leaders in efficiency (units on the verge of efficiency) and then observe the configuration of their characteristics, methods and management techniques and creating a set of recommendations to be adopted by inefficient units. For this project, this means observing the companies with the highest efficiency of high-performance businesses. The DEA model set to input was used in the study, because the effort is aimed at creating positive characteristics of high efficiency.

All the surveyed firms were high-performance organizations, taking into account their financial and non-financial performance compared with an average efficiency of the industry. However, in the DEA model, not all of them were on the verge of efficiency, which is not surprising, because even amongst highperformance companies there must be companies with higher and lower efficiency. The main advantage of the DEA method is to identify the former; further analysis will focus on their characteristics and their configurations. Four companies were on the border of effectiveness and they have efficiency ratio equal to 1 , for the rest this ratio varies between 0.45 and 0.85 . To meet the needs of our research, the extreme efficiency companies were encoded as APS, DPS, SBS, and MGS. They form a basis for the analysis of positive characteristics of high efficiency. Inputs and outputs for the four companies are presented in Table 2. 
THE POSITIVE POTENTIAL

Przemysław Zbierowski

Table 2.

Study results

Source: own study.

\begin{tabular}{|l|c|c|c|c|}
\hline \multicolumn{1}{|c|}{ Code } & APS & DPS & SBS & MGS \\
\hline Sales growth (\%) & 80.7 & 26 & 20.74 & 23.19 \\
\hline Income / employment (thousand zloty) & 42.2 & 50.34 & 56.81 & 30.04 \\
\hline Self-assessment of efficiency & 4 & 4 & 4 & 4 \\
\hline Job satisfaction & 4.33 & 3.67 & 6 & 6.33 \\
\hline Reliability & 3.33 & 4 & 4 & 4 \\
\hline Innovation & 5.33 & 4.33 & 6 & 2.67 \\
\hline Excellence of vision & 5.33 & 5.67 & 6 & 7 \\
\hline Work commitment & 4.4 & 4.6 & 4 & 6.8 \\
\hline Organizational dedication & 5.75 & 5 & 4.5 & 6 \\
\hline Empowerment & 5.83 & 6.25 & 5.67 & 5.33 \\
\hline Functional flexibility & 5.6 & 5.8 & 5 & 6.6 \\
\hline Decentralization and Deformalization & 4 & 2.25 & 3 & 1.5 \\
\hline Openness of communication & 5.5 & 5 & 5 & 5 \\
\hline Social integration & 4.89 & 5.33 & 5.56 & 6 \\
\hline Knowledge sharing & 5.25 & 5.5 & 6 & 5.25 \\
\hline Trust & 5.5 & 5.75 & 6 & 6 \\
\hline Justice & 6.33 & 5.67 & 6 & 6.33 \\
\hline Improvisation & 5.45 & 4.44 & 5.33 & 5.22 \\
\hline Internal motivation & 5.17 & 6 & 5.67 & 5.83 \\
\hline
\end{tabular}

Leaders of the effectiveness employ 10 to 25 people. Their sales range between 15 and $43 \mathrm{~m}$ zloty, and profit between 420 and 1,250 thousand zloty. Leaders of efficiency reached sales growth of 21 per cent to 81 per cent during the year preceding the survey. Each of the surveyed companies has slightly different profile of both effectiveness and positive characteristics of high efficiency. Interestingly, companies that are highly efficient in terms of profit (SBS) were somewhat less effective in terms of growth, and rapidly growing company (APS $-81 \%$ growth in the year preceding the survey) were slightly less profitable. Identification of both types of companies as leaders in efficiency was possible through the use of data envelopment analysis that takes into account a number of inputs and results of operations. All leaders of efficiency do not see themselves as such; they value their performance at 4 on a 5-point scale. Leaders of efficiency vary considerably in terms of employee satisfaction ( 3.67 to 6.33 on a 7-point scale) and innovation ( 2.67 to 5.33 on a 7 -point scale).

Similar differences as to the results of the DEA model for inputs can be observed. Generally one can say that all leaders of efficiency achieve high levels for most of the positive characteristics of high efficiency, but there are some exceptions. 
Three of the four leaders in efficiency are characterized by only an average level of involvement in the work of their employees (4 to 4.6 on a 7-point scale) (except MGS - 6.8). An interesting contradiction can be observed for the flexibility of the structure. All leaders of efficiency achieve high functional flexibility (5 to 6.6 on a 7-point scale), but low or average flexibility as decentralization or deformalization (1.5 to 4 on a 7-point scale). Ability to function in situations of stress and pressure as one of the dimensions of improvisation reaches a low level to medium (2.33 to 4.67 on a 7-point scale) for all firms, but the cumulative level of improvisation is rather high (5.22 to 5.45 on a 7-point scale) (in addition to DPS - 4.44). For the rest of positive characteristics of high efficiency all companies achieved a high level: excellence of vision (5.33 to 7 on a 7 -point scale), organizational commitment ( 4.5 to 6 on a 7 -point scale), empowerment (5.33 to 6.25 on a 7 -point scale), openness of communication ( 5 to 5.5 on a 7-point scale), social integration (4.89 to 6 on a 7-point scale), knowledge sharing (5.25 to 6 on 7-point scale), trust ( 5.5 to 6 on a 7-point scale), perceived fairness (5.67 to 6.33 on a 7-point scale), and internal motivation (5.17 to 5.83 on a 7-point scale).

These results provide partial support for the H1 hypothesis. Most of the positive characteristics of high efficiency reach a high level of efficiency for all leaders of efficiency, except for cases where it seems national culture, the size of organization and other characteristics have an important influence. However, the results do not support the $\mathrm{H} 2$ hypothesis - not all positive characteristics of high efficiency achieve high levels for all organizations. We can therefore conclude that there are different profiles of efficiency, thus there is no such thing as a universal configuration of positive characteristics always leading to high efficiency.

\section{Discussion on results}

The results of this study indicate that most of the positive characteristics of high efficiency are common for leaders. These are: excellent vision, organizational commitment, empowerment, open communication, knowledge sharing, social integration, high level of confidence, perceived justice, general improvisation, functional flexibility and internal motivation. However, some of the proposed characteristics do not reach a high level for all companies. They are: employee involvement in work, decentralization and deformalization, and ability to work in an environment of stress and pressure. The reason for low level of commitment to work may lie in the specific nature of Polish national culture in which the companies operate. The measurement of work commitment was designed for Anglo-Saxon conditions where the very commitment to work is part of the Protestant work ethic, which is quite exotic in Central Europe, where the family and private life are much more important values than the attachment to work. Low level of decentralization and deformalization can be relatively easily explained by the size of the surveyed companies. Although companies of different sizes

THE POSITIVE POTENTIAL

Przemysław Zbierowski 
THE POSITIVE POTENTIAL

Przemysław Zbierowski were tested, only the small organizations were on the verge of efficiency. In small companies, most power rests in the hands of the owner-manager, so there is little pressure for decentralization. If, however, we examine deformalization separately, it turns out that its level is average, but higher than decentralization. However, it can turn out that this level of deformalization is the highest possible given the Polish business environment, which enforces application of many administrative procedures.

Profiles efficiency leaders differ, but it should be noted that all of them managed to balance the short-term and long-term effectiveness. Also, the manner in which achieve high efficiency is different, though, that they act in the same environment and in the same industry, which generally was profitable at the time of the study and offered an opportunity for high efficiency. APS is a company that achieved the largest sales increase (81\%). It is slightly less profitable, but it has a high level of innovation. It is also characterized by a higher than average level of justice, and decentralization and deformalization. DPS makes high profit, but only an average growth and innovation. It is a leader of empowerment and selfmotivation, but it achieves only an average job satisfaction. SBS is a company with the highest profit per employee, but with the lowest growth. It is also high in terms of job satisfaction and innovation. Given the investment model, SBS has the highest level of knowledge sharing, resulting in innovation and trust. MGS is a company with the lowest profitability and innovation, but the highest employee satisfaction. Interestingly, is also characterized by the highest excellence of vision, commitment to work, functional flexibility and social integration.

It is noteworthy that all the respondents of efficiency leaders rated their performance as high, but not very high (4 on a 5-point scale). Also, the assessment of reliability was average or high $(3.33-4$ in a 5-point scale), but not very high. This is contrary to objective data on financial performance and reception of these companies by the market, which was very positive. A possible explanation is that the effectiveness leaders have even higher aspirations regarding the effectiveness and credibility.

\section{Limitations and future directions of research, practical recommendations}

There are some limitations of the presented research. First, they focus only on companies from one industry. It is a requirement of the DEA, however, it causes the risk of generalization of the results of such research. In another industry and environment other configuration of characteristics may be necessary to achieve high efficiency. The generalization is also limited by the very method, the data envelopment analysis, which is not a method of statistical data analysis, and it is more aimed at proposing practical recommendations than building a theory. Another limitation is the assessment size - it simplifies the model and makes it impossible to analyze the relationship and cause and effect relationships without 
the support of other quantitative or qualitative methods. Under these conditions, only the overall impact of configuration of expenditures on the configuration of results may be subject to examination by DEA. Also, the assessment size is a result of requirements of data envelopment analysis of high homogeneity of the assessment. The measurement of the positive characteristics of high performance seems to be another limitation of the study. Some of the measures and tools have been developed on an individual or team level and then adapted to measure at the organizational level, which may not provide fully reliable measurements.

Future research directions in this field should be related to the limitations of the study. There is a need to create fully reliable measurement tools for positive phenomena. In future studies there should be used numerous assessments of homogeneous organizations, in order to allow the use of additional quantitative analysis, to examine the relationship between individual characteristics and measures of the effectiveness of the organization, thus building a theory based on tests carried out. To generalize the results of studies comparing the number of branches seems to be a good direction for future research. Finally, the use of qualitative methods of analysis should result in a fuller understanding of the complexity of the positive characteristics of high efficiency.

The presented findings have implications for theory and research, business practices and education. More than concrete results, the theoretical idea of combining positive science of the organization with the theory of high-performance organization is a valuable contribution and starting point for future exploration of high-efficiency model. A set of recommendations for entrepreneurs and managers in the direction of achieving high efficiency can be extracted from the presented results. It includes the creation and promotion of excellent vision, strengthening the staff, support for functional flexibility and knowledge sharing, enabling open communication, creating a high level of trust and justice, recruiting internally motivated employees. One can also offer tips for teaching to include positive phenomena in curricula as factors supporting high efficiency. The presented study is the first attempt at studying the effectiveness of small and medium businesses and the impact on it of positive phenomena using data envelopment analysis. Focusing only on high-efficiency and taking into account positive phenomena gives us a valuable insight into the use by small and medium-sized companies of different profiles of positive behaviour to achieve high-efficiency also for other profiles that differ from each other. On the other hand, it is clear that there is a need to verify the model of positive characteristics of high efficiency, and prove its credibility.

\section{References}

Cameron, K.S., Dutton, J.E., \& Quinn, R.E. (2003), Positive Organizational Scholarship. Foundations of a new discipline, Berrett-Koehler Publishers, San Francisco.
THE POSITIVE POTENTIAL

Przemysław Zbierowski 
THE POSITIVE

POTENTIAL

Przemysław Zbierowski
Cameron, K.S., Dutton, J.E., Quinn, R.E., \& Wrzesniewski, A. (2003), "Developing a discipline of positive organizational scholarship”, in: Cameron, K.S., Dutton, J.E., Quinn, R.E. (Eds.), Positive Organizational Scholarship. Foundations of a new discipline, Berrett-Koehler Publishers, San Francisco.

Cooperrider, D.L., Srivastava, S. (1987), Appreciative inquiry in organizational life, JAI Press, Greenwich.

De Waal, A.A. (2005), The characteristics of a high performance organization, British Academy of Management, Oxford.

De Waal, A.A. (2006), Strategic performance management. A managerial and behavioral approach, Palgrave/Macmillan, Hampshire/New York.

Galbraith, J.R. (1995), Designing organizations: An executive briefing on strategy, structure, and process, Jossey-Bass, San Francisco.

Holbeche, L. (2005), The high performance organization. Creating dynamic stability and sustainable success, Elsevier/Butherworth Heinemann, Oxford.

Light, P.C. (2005), The four pillars of high performance. How robust organizations achieve extraordinary results, McGraw-Hill, New York.

Seligman, M.E. (2002), Authentic happiness. Using the new positive psychology to realize your potential for lasting fulfillment, Free Press, New York.

Stankiewicz, M.J. (Ed.) (2010), Pozytywny Potencjał Organizacji. Wstęp do użytecznej teorii zarządzania, Wydawnictwo TNOiK „Dom Organizatora”, Toruń. 